\title{
Résultats De La Prise En Charge Des Urgences Chirurgicales Coliques Au Centre Hospitalier Universitaire Régional De Ouahigouya, Burkina Faso
}

\author{
Jl Kambire Jl., \\ Oueddraogo S., \\ Ouedraogo S., \\ Bere B., \\ Service de chirurgie, Centre Hospitalier \\ Universitaire Régional de Ouahigouya, Burkina Faso
}

Zida M.,

Service de chirurgie,

Centre Hospitalier Universitaire Yalgado Ouédraogo, Burkina Faso

Doi:10.19044/esj.2020.v16n15p173 URL:http://dx.doi.org/10.19044/esj.2020.v16n15p173

Résumé

But : Le but de cette étude était de déterminer les résultats de la prise en charge des lésions coliques au cours des urgences chirurgicales digestives. Patients et Méthode : il s'agissait d'une étude transversale descriptive sur 12 mois, réalisée de manière rétrospective entre le $1^{\mathrm{er}}$ janvier et le 31 décembre 2016 au centre hospitalier universitaire régional de Ouahigouya. Elle avait permis de colliger 34 cas de patients opérés pour une urgence chirurgicale colique présentant un syndrome occlusif ou un syndrome péritonéal et ayant un dossier clinique complet. Résultats : Les lésions coliques avaient représenté $8,65 \%$ des urgences chirurgicales digestives. Vingt-cinq patients étaient de sexe masculin et 9 de sexe féminin, soit un sex-ratio de 2,77. La moyenne d'âge était de 37 ans. Les principales étiologies étaient les volvulus coliques dans $35,3 \%$ et les tumeurs coliques dans $32,4 \%$. Le traitement chirurgical était fonction de la lésion retrouvée en peropératoire. Les résections coliques avec anastomose immédiate avaient représenté $67,6 \%$ des cas. Les suites opératoires avaient été simples dans 67,64\%. Des complications post opératoires étaient notées dans 11 cas, soit une morbidité globale de 32,3\%. La mortalité globale était de 23,5\%. Conclusion : Les lésions coliques sont relativement fréquentes. Leurs étiologies restent dominées par les volvulus et les tumeurs coliques. Leur prise en charge est grevée d'une lourde mortalité imputable en grande partie à la nécrose intestinale, conséquence du retard diagnostique. L'amélioration du pronostic 
de ces lésions coliques repose sur une prise en charge précoce et adéquate des patients.

Mots-clés : Urgences Chirurgicales Coliques- Volvulus -Mortalité Élevée, Burkina Faso

\title{
Results of the Management of Colonic Surgical Emergencies at the Regional University Hospital Center of Ouahigouya, Burkina Faso
}

\author{
Jl Kambire Jl., \\ Oueddraogo S., \\ Ouedraogo $S$., \\ Bere B., \\ Service de chirurgie, Centre Hospitalier \\ Universitaire Régional de Ouahigouya, Burkina Faso \\ Zida M., \\ Service de chirurgie, \\ Centre Hospitalier Universitaire Yalgado Ouédraogo, Burkina Faso
}

\begin{abstract}
Aim: The aim of this study was to determine the results of the management of colonic lesions during digestive surgical emergencies. Patients and Method: This was a descriptive cross-sectional study over 12 months, carried out retrospectively between January 1 and December 31, 2016 at the regional university hospital center of Ouahigouya. It made it possible to collect 34 cases of patients operated on for a colonic surgical emergency with occlusive syndrome or peritoneal syndrome and having a complete clinical record. Results: Colonic lesions represented $8.65 \%$ of digestive surgical emergencies. Twenty-five patients were male and 9 female, for a sex ratio of 2.77. The average age was 37 years old. The main etiologies were dominated by colonic volvulus in $35.3 \%$ and colonic tumors in $32.4 \%$. Surgical treatment was based on the lesion found intraoperatively. Colon resections with immediate anastomosis represented $67.6 \%$ of cases. The postoperative operations were simple in $67.64 \%$. Postoperative complications were noted in 11 cases, representing an overall morbidity of $32.3 \%$. The overall mortality was $23.5 \%$. Conclusion: Colonic lesions are relatively frequent. Their
\end{abstract}


etiologies remain dominated by volvulus and colonic tumors. Their management is burdened with a heavy mortality largely due to intestinal necrosis, consequence of the diagnostic delay. Improving the prognosis of these colonic lesions is based on early and adequate patient management.

Keywords: Colonic Surgical Emergencies - Volvulus - High Mortality, Burkina Faso

\section{Introduction}

Les urgences chirurgicales désignent des affections, qui pour la plupart, faute d'une intervention chirurgicale obtenue sans délai, font succomber les malades en quelques heures ou en peu de jours (Mondor, 1974). Ces urgences chirurgicales sont fréquentes et peuvent intéresser tous les organes du tube digestif. Les lésions coliques, sans être les plus fréquentes, sont redoutables. Elles relèvent de plusieurs étiologies. Si dans les pays du Nord, la maladie diverticulaire et les tumeurs coliques prédominent, les volvulus coliques sont la première étiologie dans les séries africaines. Ils représentaient 5,4\% au Niger (Harouna et al, 2001). La prise en charge en urgence des lésions coliques reste grevée d'une lourde mortalité, $16,5 \%$ en Côte d'Ivoire (Yenon et al, 2008). La présente étude a pour but de déterminer les étiologies et les résultats de la prise en charge des urgences chirurgicales coliques au centre hospitalier universitaire régional de Ouahigouya.

\section{Patients et Méthode}

Il s'agissait d'une étude transversale descriptive dont la collecte de données avait été réalisée de manière rétrospective au centre hospitalier universitaire régional de Ouahigouya entre le $1^{\text {er }}$ janvier 2016 et le 31 décembre 2016. Elle avait concerné tous les patients opérés pour une urgence chirurgicale colique durant la période d'étude présentant un syndrome occlusif ou un syndrome péritonéal et possédant un dossier clinique bien documenté. Les variables étudiées étaient l'âge, le sexe, le délai de consultation, l'étiologie, le traitement et les modalités évolutives. N'avaient pas été inclus, les patients chez qui le dossier clinique était manquant ou insuffisamment renseigné. Les résultats étaient présentés sous forme de moyenne ou de pourcentage.

\section{Résultats}

Au cours de notre période d'étude, 394 patients avaient été opérés pour une urgence chirurgicale digestive dont 34 pour une urgence chirurgicale colique, soit une fréquence globale de $8,62 \%$ des urgences chirurgicales digestives. La moyenne d'âge des patients était de 37 ans avec des extrêmes de 7 mois et 80 ans. Vingt-cinq patients $(73,5 \%)$ étaient de sexe masculin et 
neuf $(26,5 \%)$ étaient de sexe féminin, soit un sex-ratio de 2,77. Concernant le délai diagnostique cinq patients $(14,7 \%)$ étaient admis dans le service dans les 24 premières heures suivant le début de leur maladie ; 18 (52,9\%) l'avaient été entre le $2^{\text {ème }}$ et le $4^{\text {ème }}$ jour et 11 patients $(32,4 \%)$ avaient été admis à partir du $5^{\text {ème }}$ jour. Le tableau clinique était dominé par les douleurs abdominales retrouvées chez tous les patients. Les vomissements étaient notés dans 15 cas $(44,1 \%)$ et l'arrêt des matières et des gaz dans 17 cas (50\%). Aucune exploration radiologique n'avait été réalisée. L'indication opératoire avait été retenue sur la base de l'existence d'un syndrome occlusif ou d'un syndrome péritonéal. Le diagnostic avait été peropératoire. Les étiologies des lésions coliques au cours des urgences chirurgicales digestives avaient été multiples. Elles avaient été dominées par les volvulus coliques dans 12 cas $(35,3 \%)$ et les tumeurs coliques dans 11 cas $(32,4 \%)$. Les différentes étiologies sont répertoriées dans le tableau $\mathrm{I}$.

Tableau I : Récapitulatif des étiologies des lésions coliques au cours des urgences chirurgicales

\begin{tabular}{c|cc}
\multicolumn{1}{c}{ Etiologies } & Nombre de de cas & Pourcentage (\%) \\
\hline Volvulus & 12 & 35,3 \\
Tumeurs & 11 & 32,3 \\
Invaginations & 08 & 23,5 \\
Traumatismes & 03 & 8,8 \\
Total & 34 & 100
\end{tabular}

Tous les patients avaient bénéficié d'une réanimation préopératoire. Cette réanimation avait été poursuivie en per opératoire et en post opératoire. Un traitement antalgique à base de paracétamol avait été systématique. Il avait été associé au néfopam injectable chez le sujet adulte. Une bi-antibiothérapie à base de céphalosporine de $3^{\text {ème }}$ génération et de métronidazole avait été associée. Le traitement chirurgical avait été réalisé par laparotomie. A l'ouverture, une nécrose intestinale avait été notée dans sept cas (20,6\%). Les gestes chirurgicaux réalisés avaient été fonction de l'étiologie. Ces gestes avaient été dominés par la colectomie idéale segmentaire gauche dans 15 cas $(44,11 \%)$ et l'hémicolectomie droite avec rétablissement immédiat de la continuité dans huit cas $(23,5 \%)$. Le tableau $\mathrm{N}^{\circ} \mathrm{II}$ avait résumé le geste chirurgical réalisé en fonction de l'étiologie. 
Tableau II : Récapitulatif des gestes chirurgicaux réalisés en fonction des étiologies et de leurs sièges

\begin{tabular}{|c|c|c|c|c|}
\hline Etiologies & $\begin{array}{l}\text { Type } \\
\text { anatomique }\end{array}$ & Geste chirurgical & Nombre & $\begin{array}{l}\text { Taux } \\
(\%)\end{array}$ \\
\hline \multirow{5}{*}{ VOLVULUS } & \multirow[b]{2}{*}{ sigmoïde } & \multirow{2}{*}{$\begin{array}{l}\text { Colectomie idéale } \\
\text { Désinvagination et } \\
\text { pexie }\end{array}$} & 8 & 23,53 \\
\hline & & & 1 & 2,94 \\
\hline & coecum & $\begin{array}{l}\text { Hémicolectomie } \\
\text { droite et anastomose }\end{array}$ & 1 & 2,94 \\
\hline & $\begin{array}{l}\text { Nœud } \quad \text { iléo } \\
\text { sigmoïdien }\end{array}$ & $\begin{array}{l}\text { Double résection et } \\
\text { anastomose }\end{array}$ & 2 & 5,88 \\
\hline & coecum & $\begin{array}{l}\text { Hémicolectomie } \\
\text { droite et anastomose }\end{array}$ & 3 & 8,82 \\
\hline \multirow{4}{*}{ TUMEURS } & & $\begin{array}{l}\text { Hémicolectomie } \\
\text { droite et iléostomie }\end{array}$ & 2 & 5,88 \\
\hline & Colon droit & $\begin{array}{l}\text { Hémicolectomie } \\
\text { droite et anastomose }\end{array}$ & 2 & 5,88 \\
\hline & Sigmoïde & Colectomie idéale & 3 & 8,82 \\
\hline & transverse & $\begin{array}{l}\text { Hémicolectomie } \\
\text { élargie } \\
\text { anastomose }\end{array}$ & 1 & 2,94 \\
\hline \multirow{3}{*}{ INVAGINATIONS } & iléocaecocolique & $\begin{array}{l}\text { Désinvagination et } \\
\text { appendicectomie }\end{array}$ & 5 & 14,7 \\
\hline & \multirow[t]{2}{*}{ Colo-colique } & $\begin{array}{l}\text { Désinvagination et } \\
\text { appendicectomie }\end{array}$ & 1 & 2,94 \\
\hline & & $\begin{array}{l}\text { Colectomie et } \\
\text { anastomose }\end{array}$ & 1 & 2,94 \\
\hline \multirow[b]{2}{*}{ TRAUMATISMES } & $\begin{array}{l}\text { Perforations } \\
\text { caecales }\end{array}$ & Excisions-sutures & 2 & 5,88 \\
\hline & $\begin{array}{l}\text { Perforations } \\
\text { multiples } \\
\text { coliques gauches }\end{array}$ & $\begin{array}{l}\text { Colectomie } \\
\text { segmentaire idéale }\end{array}$ & 1 & 2,94 \\
\hline
\end{tabular}

La durée moyenne d'hospitalisation a été de 7 jours. Les suites opératoires ont été simples chez 23 patients, soit $67,64 \%$. Des complications post opératoires ont été notées chez 11 patients, soit un taux de morbidité de $32,3 \%$. Parmi ces complications, il a été noté un choc septique dans sept cas, une infection du site opératoire dans deux cas, une fistule stercorale dans un cas et des troubles psychotiques aigus en rapport avec une stomie dans un cas. Huit décès ont été enregistrés chez les patients ayant présenté des complications, soit un taux de mortalité globale de $23,5 \%$. Parmi les patients 
décédés, une nécrose a été observée chez sept d'entre eux. Par ailleurs, une colectomie et un rétablissement immédiat de la continuité avaient été réalisés chez six d'entre eux. Chez quatre des patients décédés, on notait des âges extrêmes.

\section{Commentaires}

Dans cette série, les lésions coliques ont représenté 8,6\% des urgences chirurgicales digestives. Ce résultat est comparable aux taux de 7,6\% et de 9,4\% retrouvés au Niger (Adamou et al, 2015 ; Harouna et al, 2001). Ceci traduit l'atteinte relativement fréquente du côlon dans les affections chirurgicales digestives.

La moyenne d'âge des patients dans cette étude a été de 37 ans avec un sex-ratio de 2,77. Ces résultats diffèrent de ceux retrouvés en Côte d'Ivoire où l'âge moyen a été de 41,7 ans avec un sex-ratio de 5 (Yenon et al, 2008). Au Burkina Faso, la moyenne d'âge a été de 47,58 ans avec un sex-ratio de 1,18 (Zongo, 2012). Les résultats obtenus dans cet article pourraient s'expliquer par la structure de la population qui est à forte prédominance jeune (Ministère de la Santé du Burkina Faso, 2011) et par l'inclusion de cas pédiatriques dans l'échantillon. En dépit de ces différences de taux, le jeune âge et la prédominance masculine sont des caractéristiques communes aux séries africaines (Choua et al, 2015 ; Kouadio et al, 2003). Ceci est à l'opposé des séries occidentales qui se caractérisent par un âge plus avancé (Millat, 2003 ; Mantion et al, 2003 ; Pattriti et al, 2005). Cette différence d'avec les séries occidentales pourrait s'expliquer par la différence dans les étiologies et aussi la différence dans l'espérance de vie.

En effet, en Occident, les urgences chirurgicales coliques sont dominées par les tumeurs et la pathologie diverticulaire tandis qu'en Afrique les volvulus coliques sont au premier plan (Millat, 2003 ; Elé et al, 2006). Comme démontré ici, les volvulus coliques ont représenté la première étiologie des urgences chirurgicales coliques avec 35,3\% des cas. Il en a été de même en Côte d'Ivoire (Yenon et al, 2008) où les volvulus coliques ont représenté la première étiologie avec $56,4 \%$ des cas. L'étiopathogénie de cette pathologie incriminant des facteurs raciaux et la consommation excessive de fibres alimentaires expliquerait sa prépondérance dans les urgences coliques de cette série. Dans cet article, les tumeurs coliques ont représenté la deuxième étiologie avec 32,3\% des cas. Il en a été de même en Côte d'Ivoire (Yenon et al, 2008) où les tumeurs coliques ont occupé la deuxième place avec $29 \%$ des cas. Les résultats observés dans cette étude pourraient s'expliquer par une augmentation des cas de cancers coliques au Burkina Faso ou plutôt par une meilleure notification des cas. En effet, la fréquence hospitalière du cancer colique est passée de 1,88 cas en 2001 à 2,6 cas en 2006, puis à 4,5 cas en 2012 (Kalmogo, 2001 ; Dama 2006 ; Zongo, 2012). A l'opposé, au Niger les 
invaginations intestinales ont été prépondérantes, suivies des volvulus et des tumeurs coliques (Adamou et al, 2015). Cette situation pourrait s'expliquer par la forte proportion de cas pédiatriques dans leur série, en témoigne leur moyenne d'âge à 23 ans. Tous les patients ont bénéficié d'une prise en charge médicale et chirurgicale. La prise en charge chirurgicale a été fonction de l'étiologie.

Une laparotomie sous anesthésie générale a été réalisée chez tous les patients. Il en a été de même dans la plupart des séries africaines (Adamou et al, 2015 ; Yenon et al, 2008 ; Choua et al, 2015 ; Kouadio et al, 2003). Le traitement chirurgical a été fonction de la lésion objectivée en per opératoire. La colectomie avec rétablissement immédiat de la continuité digestive a été le geste le plus fréquemment réalisé dans cette étude avec 23 cas, soit $67,6 \%$ des cas à la différence de la série ivoirienne (Yenon et al, 2008) où la résection colique avec rétablissement différé de la continuité ont été réalisés dans 55,3\% des cas. Dans cette étude, le rétablissement immédiat de la continuité a été privilégié autant que possible du fait des problèmes liés à l'acceptabilité de la stomie par les patients mais aussi du fait de l'absence de dispositifs de stomie dans la structure où ce travail a été réalisé. Ces deux attitudes thérapeutiques sont loin de faire l'unanimité. Si pour les uns, un rétablissement immédiat de la continuité après colectomie comporte un risque plus élevé de mortalité et expose à plus de complications post opératoires à type d'infections du site opératoire et de fistules anastomotiques (Sani et al, 2003 ; Touré et al, 2003 ; Raveethiran, 2004), pour d'autres les facteurs incriminés dans la mortalité sont indépendants de la technique chirurgicale. Les facteurs tels que l'âge supérieur à 60 ans, les signes de choc et les signes de nécrose digestive seraient en cause (Atamanalp et al, 2008 ; Bhatnagar et al, 2004 ; Liang et al, 2006). Pour d'autres encore, l'indication opératoire doit être posée en fonction de l'état général du patient, de la viabilité du côlon, de sa vascularisation, de la congruence des deux extrémités et de l'expérience du chirurgien (Schwartz et al, 2009).

Dans cette étude, 11 patients ont présenté des complications postopératoires, soit un taux de morbidité de 32,3\%. Ce taux est inférieur à celui de $38,8 \%$ de la série ivoirienne (Yenon et al, 2008) ; mais il reste largement supérieur à celui enregistré au Tchad qui a été de 13,6\% (Choua et al ,2015). Le taux de mortalité dans cette étude a été de 23,5\%. Il est supérieur à ceux retrouvés en Côte d'Ivoire et au Tchad qui ont été respectivement de 16,5\% et de 4,5\% (Yenon et al, 2008 ; Choua et al ,2015). La forte mortalité dans cette étude pourrait s'expliquer par la nécrose intestinale, le choix de la technique chirurgicale et les âges extrêmes. La nécrose intestinale était présente dans $20,6 \%$ des cas de cette série; cela est dû au long délai diagnostique. En effet chez plus de la moitié des patients le diagnostic a été posé entre la quarante-huitième et la quatre-vingt-seizième heure. Ce long 
délai diagnostic est l'apanage des séries africaines (Adamou et al, 2015 ; Yenon et al, 2008; Choua et al, 2015; Kouadio et al, 2003). L'automédication, le recours premier à un traitement traditionnel, l'insuffisance du plateau technique au niveau périphérique pour le diagnostic de l'urgence chirurgicale colique et le faible accès tant géographique que financier aux structures sanitaires de référence de même que le retard accusé dans la prise en charge sont autant de facteurs qui pourraient en être la cause.

\section{Conclusion}

Les lésions coliques sont relativement fréquentes. Leurs étiologies restent dominées par les volvulus et les tumeurs coliques. Leur prise en charge est grevée d'une lourde mortalité qui pourrait être imputable à la nécrose intestinale, à la technique chirurgicale appliquée et aux âges extrêmes. L'amélioration du pronostic de ces lésions coliques requiert une prise en charge précoce mais aussi l'application d'une technique chirurgicale adéquate.

Conflit d'intérêts : Les auteurs ne déclarent aucun conflit d'intérêts.

\section{Contribution des auteurs :}

Dr KAMBIRE Jean Luc, Dr OUEDRAOGO Souleymane, Dr OUEDRAOGO Salam, Dr BERE Bernadette ont réalisé les différentes interventions et ont assuré leur suivi. Ils ont aussi assuré la recherche documentaire et la rédaction de l'article. Pr ZIDA Maurice a assuré l'encadrement scientifique.

\section{References:}

1. Mondor H. Diagnostics urgents-Abdomen, édition Masson, Paris, 1974, 1119p.

2. Harouna Y, Ali I, Séibou A et al. Deux ans de chirurgie digestive d'urgence à l'hôpital national de Niamey (Niger). Médecine d'Afrique Noire, $2001 ; 48$ (2) : 49-54.

3. Yenon KS, Lebeau R, Koffi E et al. Morbidité et mortalité postopératoire des urgences coliques non traumatiques à propos de 85 interventions. Mali Médical, 2008 ; 23 (2) : 38-42.

4. Adamou H, Amadou MM Ibrahim, Habou O et al. Retard diagnostique et implication pronostique en milieu africain. Cas des urgences en chirurgie digestive à l'hôpital national de Zinder, Niger. European Scientific Journal, 2015 ; 11 (12) : 251-262.

5. Zongo WS. Aspects épidémiologiques, cliniques paracliniques et thérapeutiques des cancers coliques en occlusion aux CHU-SS et 
CHU-YO : A propos de 36 cas. Thèse de doctorat d'état en médecine, université de Ouagadougou, 2012, n 77,145 p.

6. Ministère de la Santé du Burkina Faso. Plan National de Développement Sanitaire 2011-2020 ; 2011, 55p.

7. Choua O, Kaboro M, Ali MM Moussa et al. Résultats du traitement du volvulus du sigmoïde à N'Djaména, Tchad. European Scientific Journal, $2015 ; 11(21): 245-253$.

8. Kouadio KG, Turquin T. Cancers coliques gauches en occlusion en Côte d'Ivoire. Ann Chir, 2003 ; 128 (6) : 364-367.

9. Millat B. Traitements des cancers coliques en occlusion : chirurgie ou stents ? e-mémoires de l'Académie Nationale de Chirurgie, $2005 ; 4$ (2) :12-14.

10. Elé N, Okiémy G, Lebeau R et al. Le cancer du côlon gauche au CHU de Brazzaville. Résultats du traitement chirurgical. Mali Médical, $2006 ; 21$ (1) :1-3.

11. Mantion G, Panis Y. Mortalité et morbidité en chirurgie colorectale. Monographie de l'Association Française de Chirurgie, éditions Arnette, Paris, 2003, 224p.

12. Pattriti A, Contine A, Carbone E et al. One stage resection without colonic lavage in emergency surgery of the left colon. Colorectal Dis, 2005; 7 (4): 332-338.

13. Kalmogo E. Les cancers du côlon et du rectum au Burkina Faso, une revue de 86 cas colligés au CHU-YO et au CHU-SS. Thèse de médecine, université de Ouagadougou, 2001, 77p.

14. Sano D, Bonkoungou G, Zongo $\mathrm{N}$ et al. Occlusions coliques par cancers aux urgences viscérales du CHU-YO. Guinée Médical, 2008 ; $60: 54-58$.

15. Sani R, Ganda OR, Harouna YD et al. Traitement du volvulus du côlon sigmoïde à l'hôpital national de Niamey : à propos de 68 cas (Niger). Journal Africain de Chirurgie Digestive, 2003 ; 3 (2) : 277-280.

16. Touré CT, Dieng M, Sanou A et al. Résultats de la colectomie en urgence dans le traitement du volvulus du côlon au centre hospitalier universitaire de Dakar. Annales de chirurgie, 2003 ; 128 (2) : 98-101.

17. Raveethiran V. Restorative resection of unprepared left colon in gangrenous vs viable sigmoid volvulus. Int J Colorectal Dis, $2004 ; 19$ (3) : 258-263.

18. Atamanalap SS. Classification of sigmoid volvulus. Turk J Med Sci, 2008; 38 (5): 425-429.

19. Bhatnagar BN, Sharma CL, Gautan A. et al. Gangrenous sigmoid volvulus: a clinical study of 76 patients. Int J Colorectal Dis, 2004; 19 (2): 134-142. 
20. Liang JT, Lai HS, Lee PH. Elective laparoscopically assisted sigmoidectomy for the sigmoid volvulus. Surg Endosc, 2006; 20 (11): 1772-1773.

21. Schwartz A, Peycru T, Tardat E. et al. Prise en charge actuelle du volvulus du sigmoïde en milieu tropical. Méd Trop, 2009 ; 69 (1) : 5155. 